\title{
Collective dynamics, fluctuations and instabilities in relativistic heavy-ion collisions
}

\author{
LÁszló P. CSERnai
}

\author{
Department of Physics and Technology, \\ University of Bergen, Allegaten 55, 5007 Bergen, Norway
}

\begin{abstract}
Collective processes occurring in high energy heavy ion collisions are discussed. The observed fluctuations and asymmetries have different causes, which is shown clearly in the experimental data for peripheral collisions. We discuss how these can be separated and which are the most interesting collective instabilities, which do not arise from random statistical fluctuations.
\end{abstract}

The angular fluctuations of Cosmic Microwave Background are well studied, with the Planck satellite we observed these fluctuations with a very high resolution beyond multipole moment 1000, but the dominant peak is around 200, which means that a typical fluctuation peak is a bit larger than one degree.

The same type of study for central heavy ion collisions at the CERN LHC for the azimuth angle in the direction transverse to the beam showed a maximum for multipolarity three, or in other words the largest flow harmonics was $v_{3}$ and exceeded 0.30 [1]. It is important that these collisions were almost exactly central ones, with zero impact parameter, thus the initial condition could not lead to a collective azimuthal asymmetry. So the observed asymmetry could be originated from random fluctuations only. Studying fluctuation systematics for both central and different peripheral collisions, shows that the $v_{2}\left(p_{t}^{\text {large }}\right)$ vs. $v_{2}\left(p_{t}^{\text {low }}\right), v_{2}$ vs. $v_{3}, v_{2}$ vs. $v_{4}, v_{2}$ 
vs. $v_{5}$, show distinctly different correlations for central and peripheral collisions [2]. Random fluctuations can have several reasons, e.g. the random initial nucleon configurations, or the critical fluctuations in a phase transition at the hadronization of the Quark Gluon Plasma (QGP). If we study the shape of density or energy density distribution in a finite system during hadronization, we can see that the Kurtosis of the distribution is becoming negative during the transition (critical opalescence), while the Skewness of the distribution changes from negative to positive as the matter hadronizes starting from QGP [3]. If we compare this with the observed negative Skewness of the multipoilarity distribution in central heavy ion collisions, which is negative, this could indicate that the fluctuation arise from flow fluctuations from the QGP phase. This does not contradict with the observed constituent quark number scaling of flow harmonics of different hadrons, which also indicates that the flow harmonics should have developed mainly in the QGP phase.

If we want to analyse peripheral reactions we have to separate flow from random fluctuations and from initial global symmetries. These latter ones are correlated with the center of mass and reaction plane of the event, which can and should be determined Event by Event (EbE). This is in part already done in experiments [4], while part of the complete procedure was proposed to be performed [5].

Thus, if we know the EbE reaction plane (RP) and the center of mass $(\mathrm{CM})$ rapidity $y_{\mathrm{cm}}$, then we can analyse the both the collective and fluctuating flow separately. This separation can be done if all data are provided in the EbE RP,CM system in the format usually presented recently as if only fluctuations would exist. This way of analysis works for exact central collisions but as mentioned it is inadequate for non-central ones [2].

The way how to make this separation is given in Ref. [6] Recently flow data are parametrized in the form

$$
\begin{array}{r}
\frac{d^{3} N}{d y d p_{t} d \phi}=\frac{1}{2 \pi} \frac{d^{2} N}{d y d p_{t}}\left[1+2 v_{1}\left(y, p_{t}\right) \cos \left(\phi-\Psi_{1}^{E P}\right)+\right. \\
\left.2 v_{2}\left(y, p_{t}\right) \cos \left(2\left(\phi-\Psi_{2}^{E P}\right)\right)+\cdots\right],
\end{array}
$$

where $v_{n}$ and $\Psi_{n}^{E P}$ are measured averages for a set of events in a given impact parameter, $b$, transverse momentum, $p_{t}$, and rapidity, $y$, bin. The $\Psi_{n}^{E P}$ values should be measured with respect to a given angle in a given event, most reasonably with respect to the EbE RP angle, $\Psi_{R P}$. Similarly the rapidity variable should also be given with respect to the EbE CM rapidity, $y_{C M}$. In most publications $\Psi_{R P}$ and $y_{C M}$ are not evaluated and thus the 
$\Psi_{n}^{E P}$ values are not published. This is possible with the "cumulative" methods, which can give the absolute values of the $v_{n}$ parameters, but present a rather constrained information about the flow process.

Assuming that the complete experimental data set is given (published), $v_{n}\left(y-y_{C M}, p_{t}\right), \Psi_{n}^{E P}$, with respect to $\Psi_{R P}$, then one can decompose the flow parametrization into a fluctuating and into a collective part [6]:

$$
\begin{aligned}
v_{n \text { even }}^{\text {Coll. }} \cos \left[n\left(\phi-\Psi_{n}^{E P}\right)\right]= & \frac{1}{2}\left[{ }^{c} v_{n}\left(\mathbf{y}, p_{t}\right) \pm{ }^{c} v_{n}\left(-\mathbf{y}, p_{t}\right)\right] \cos (n \phi) \\
v_{n \frac{\text { even }}{\text { odd }}}^{\text {Fluct. }} \cos \left[n\left(\phi-\Psi_{n}^{E P}\right)\right]= & \frac{1}{2}\left[{ }^{c} v_{n}\left(\mathbf{y}, p_{t}\right) \mp{ }^{c} v_{n}\left(-\mathbf{y}, p_{t}\right)\right] \cos (n \phi) \\
& +{ }^{s} v_{n}\left(\mathbf{y}, p_{t}\right) \sin (n \phi)
\end{aligned}
$$

where $\mathbf{y}=y-y_{C M}$, and new coefficients

$$
{ }^{c} v_{n} \equiv v_{n} \cos \left(n\left(\Psi_{n}^{E P}\right)\right) \text { and }{ }^{s} v_{n} \equiv v_{n} \sin \left(n\left(\Psi_{n}^{E P}\right)\right) \text {, }
$$

are constructed, so that ${ }^{s} v_{n}$ can be due to fluctuations only and the Colelctive part can have only azimuthal angle dependence $\cos (n \phi)$ because of the mirror symmetry accross the RP.

We can this way see that the Collective flow components are such that these have cosine azimutal dependence only and the even harmonics are rapidity even functions, while the odd harmonics are rapidity odd functions. The fluctuating flow components have both sine and cosine azimuthal dependence. This separation of the flow enables us to discuss and analyze the two types of flow processes separately. On should also anticipate that for the Fluctuationg flow component the sine and cosine components in the flow should be of similar magnitude.

This separation is important for analysing both fluctuations and collective flow in peripheral collisions. For example viscosity usually damps flow structure and asymmetries, while critical fluctuations may build up with time. Thus, to evaluate viscosity with precision, requires the separation of collective and fluctuating flows. Initial random fluctuations are usually damped with time due to viscosity. On the other hand initial shear flow can feed the development of Kelvin Helmholtz Instability, thus increasing rotation and turbulence. For converting shear flow into rotation and KHI som, (but low) viscosity is required.

Recently there is an increasing theoretical effort to study the effects of increasing angular momentum with increasing beam energy in peripheral collisions [7-15]. Here the initial shear plays an important role. The development of strong fields (e.g. Color Glass Condensate, CGC) leads to a more 
compact and more energetic initial state, and streak by streak momentum conservation leads to strong shear. Still in simplified models the matter is assumed to propagate to infinity without hindrance, leading to a uniform Bjorken type flow, without shear. Thus, the realistic assumptions on the initial state are vital in getting a realistic view about the collision process. If initial shear is neglected, vorticity is not formed and no turbulence or KHI could develop.

The initial shear leads to a much larger ( $3 \mathrm{c} / \mathrm{fm}$ ) vorticity [16] than random fluctuations ( $0.2 \mathrm{c} / \mathrm{fm})$ [17]. This can lead to different observable effects: Two particle correlations or particle polarizatons. The large vorticity, i.e. local orbital motion equilibrates with the spins of the constituent particles of matter due to the mechanical equipartition principle [18]. The arising $\Lambda$ polarization is evaluated [19] and it was obtained that for particles emitted in the reaction plane, transverse to the beam ( $x$-direction) one can observe a transverse $(-y$-directed) polarization of 8-9\%. In other azimutal directions the polarization vanishes.

Although, this was measured in an experiment already in 2007 [20], all emission angles for all azimuth were averaged over, and the EbE CM and the RP were not precisely determined. One has to emphasize that for the polarization measurements the Projectile and Target side must be determined otherwise this effect obviously vanishes. So the usual cumulative methods are not adequate at all for these measurements.

Another important consequence of this mechanical polarization that being a paramagnetic type of effect, with high temperature the polarization decreases. Thus, the characteristic quantity is the thermal vorticity $\nabla \times \boldsymbol{\beta}$ where,

$$
\beta^{\mu}=u^{\nu}(x) / T(x),
$$

i.e. the flow velocity is divided by the temperature. This is important because although the angular momentum and vorticity increase with increasing beam energy the freeze-out temperature is also higher. Thus, it turned out that the polarization is somewhat larger at RHIC energies than at the LHC, furthermore still ar NICA and FAIR energies one can obtain significant thermal vorticity [21].

It was also shown that the Hanbury Brown and Twiss effect is aslo sensitive on rotation [22], and interestingly the azimuthal asymmetry of the source as well as the rotation of the source may interfere with each other, so one has to find the adequate azimuth angle where one can measure exclusively the rotation.

The interest in rotation in high energy heavy ion collisions led to the 
development of an Exact expanding and rotating fluid dynamical model [23]. The model parameters were compared to the fluid dynamical model of a peripheral collision, and the initial sizes and angular and expansion velocities were determind to obtain a best fit to the realistic fluid dynamical solutions [24]. In this model the vorticity was evaluated [25], the arising two particle correlations [26], as well as the arising polarization [27].

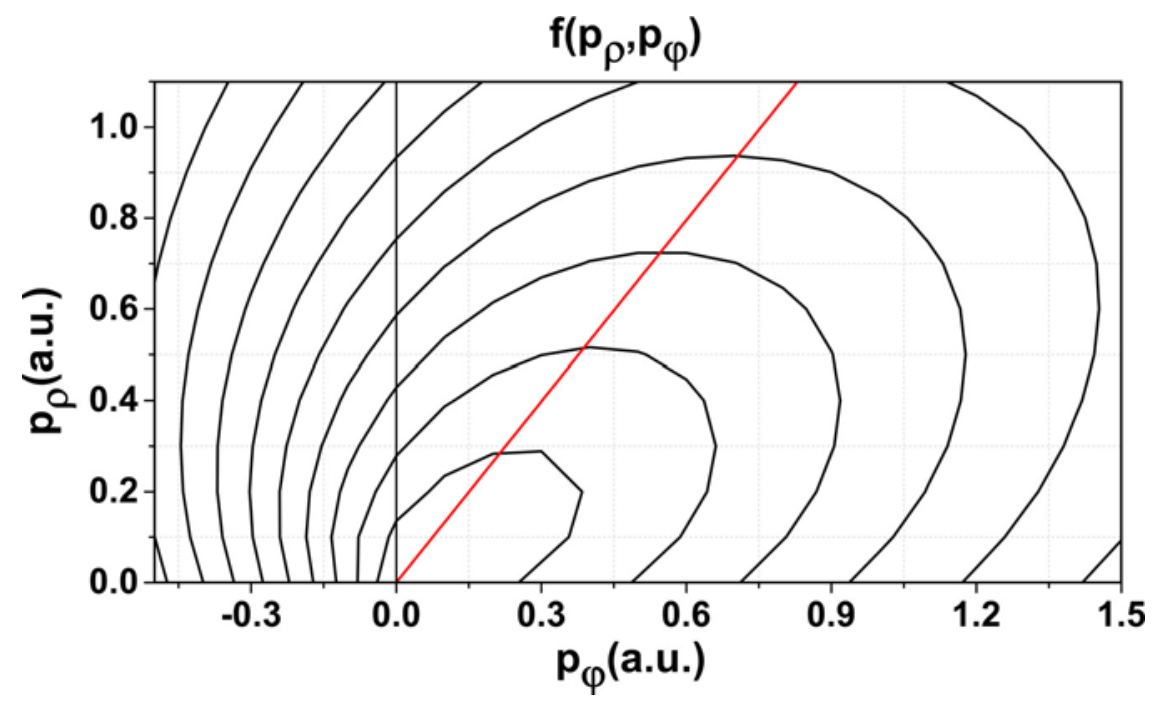

Figure 1: The schematic phase space distribution of the rotating and expanding source in the momentum space. The momentum of the expansion increases with the radius just as the momentum arising from the rotation. So, higher radial flow momenta correspond to higher rotation momenta, indicated by the red line. The distribution is thermally smeared as indicated by the contour lines. From Ref. [26].

It is interesting that the two particle correlations also indicate the rotation of the system. As pointed out in [26] the expanding and rotating sustem is azimuthally symmetric in the configuration space, but not in the momentum space as shown in Fig. 1. The momentum of the fluid motion is characterized by the flow velocity, this is indicated by the red full line in Fig. 1. This characteristic flow velocity distribution determines the final momentum distribution of the emitted particles.

The results of the latest work $[27,28]$, are discussed in the contribution of Yilong Xie, which can be found in this proceedings. These results included the evatuation of the polarization arising from the time derivative of the thermal vorticty, which is turned out to be not negligible. These results were also compared to the fluid dynamical model result of the Itanian ECHOQGP code [11]. 
Thus, both fluctuations and collective flow processes carry important information on the dynamics of the system and thus on the propoerties of QGP. For a precise analysis, forst of all the fluctuatinf and collective flow components should be separated and for this we need the accurate determination of the EbE collision symmetry axes, the Reaction plane and the Center of mass.

The most sensitive measurement of viscosity can be achieved by phenomena which are directly dependent on the shear viscosity like the Kelvin Helmholtz Instability. To study these processes high resolution relativistic fluid dynamical codes are required, which can handle shear and turbulence. Necessarily, the initial shear cannot be neglected if we intend to study the shear viscosity.

\section{References}

[1] K. Aamodt et al. (ALICE Collaboration), Phys. Rev. Lett. 107, 032301 (2011), and CERN Courier, October 2011, p. 6.

[2] Michael Weber (CERN) talk (ATLAS data) at the "X Workshop on Particle Correlations and Femtoscopy WPCF 2014", 25-29.08.2014, Kroly Rbert College, Gyngys, Hungary.

[3] L.P. Csernai, G. Mocanu, Z. Neda, Phys. Rev. C 85, 068201 (2012).

[4] R. Snellings, J. Phys. G 41, 24007 (2014).

[5] L.P. Csernai, G. Eyyubova, V.K. Magas, Phys. Rev. C 86, 024912 (2012).

[6] L.P. Csernai, H. Stöcker, J. Phys. G 41, 124001 (2014).

[7] L.P. Csernai, V.K. Magas, H. Stöcker, D.D. Strottman, Phys. Rev. C 84, 024914 (2011).

[8] L.P. Csernai, D.D. Strottman, Cs. Anderlik, Phys. Rev. C 84, 054901 (2012).

[9] D.J. Wang, Z. Néda, and L.P. Csernai, Phys. Rev. C 87, 024908 (2013).

[10] G. Graef, M. Bleicher, M. Lisa, Phys. Rev. C 89, 014903 (2014).

[11] F. Becattini, G. Inghirami, V. Rolando, A. Beraudo, L. Del Zanna, A. De Pace, M. Nardi, G. Pagliara, and V. Chandra, arXiv:1501.04468v2 [nucl-th] (2015). 
[12] Guangyao Chen, Rainer J. Fries, Phys. Lett. B 723417 (2013).

[13] G. Chen, R.J. Fries, J.I. Kapusta, Y. Li, arXiv:1507.03524v1 [nucl-th]

[14] Brett McInnes, Yen Chin Ong, Nucl. Phys. B 898, 197 (2015), arXiv:1504.07344 [hep-th].

[15] Brett McInnes Nucl. Phys. B 887, 246 (2014), arXiv:1403.3258 [hep-th].

[16] L.P. Csernai, V.K. Magas, D.J. Wang, Phys. Rev. C 87, 034906 (2013).

[17] S. Floerchinger, U.A. Wiedemann, JHEP 100,1111 (2011); J. Phys. G 38,124171 (2011).

[18] F. Becattini, V. Chandra, L. Del Zanna, and E. Grossi, Ann. Phys. 338, 32 (2013); arXiv: 1303.3431 [nucl-th].

[19] F. Becattini, L.P. Csernai, D.J. Wang, Phys. Rev. C 88, 034905 (2013).

[20] B.I. Abelev et al., (STAR), Phys. Rev. C 76, 024915 (2007).

[21] L.P. Csernai, D.J. Wang, M. Bleicher, H. Stöcker, Phys. Rev. C 90, 021904(R) (2014).

[22] L.P. Csernai, S. Velle, D.J. Wang, Phys. Rev. C 89, 034916 (2014).

[23] T. Csörgő and M.I. Nagy, Phys. Rev. C 89, 044901 (2014).

[24] L.P. Csernai, D.J. Wang, and T. Csörgő Phys. Rev. C 90, 024901 (2014).

[25] L.P. Csernai, J.H. Inderhaug, Int. J. Modern Physics E 24, 1550013 (2015), arXiv:1503.03247v1 [nucl-th].

[26] S. Velle, L.P. Csernai, Phys. Rev. C 92, 024905 (2015).

[27] Y.L. Xie, R.C. Glastad, L.P. Csernai, arXiv: 1505.07221 [nucl-th].

[28] Yilong Xie, Contribution to this volume (2015). 